\title{
Volatile Organic Compound Based Markers for the Aroma Trait of Rice Grain
}

\author{
David Ocan ${ }^{1,4}$, Zhang Rongrong ${ }^{2}$, Martin Odoch ${ }^{4}$, Ephraim Nuwamanya ${ }^{3}$, Angele P. Ibanda ${ }^{1}$, Thomas L. Odong ${ }^{1}$, \\ Jimmy Lamo ${ }^{3}$, Anne M. Fitzgerald ${ }^{2}$, Venea D. Daygon ${ }^{2}$ \& Patrick R. Rubaihayo ${ }^{1}$ \\ ${ }^{1}$ School of Agricultural Sciences, Makerere University, Kampala, Uganda \\ ${ }^{2}$ School of Agriculture and Food Sciences, University of Queensland, Queensland, Australia \\ ${ }^{3}$ Cereal Programme, National Crops Resources Research Institute, Wakiso, Uganda \\ ${ }^{4}$ Faculty of Agriculture and Environment, Gulu University, Gulu, Uganda \\ Correspondence: David Ocan, Faculty of Agriculture and Environment, Gulu University, Gulu, Uganda. Tel: \\ 256-772-323-464. Fax: 256-471-32094. E-mail: d.ocan@gu.ac.ug
}

Received: May 16, 2020

doi:10.5539/jas.v12n8p92
Accepted: June 20, $2020 \quad$ Online Published: July 15, 2020

URL: https://doi.org/10.5539/jas.v12n8p92

The research is financed by Makerere University-Swedish International Development Agency (SIDA) bilateral programme and the School of Agriculture and Food Sciences, University of Queensland, Australia.

\begin{abstract}
A study was conducted to determine the volatile organic compounds (VOCs) associated with rice grain aroma in 37 commonly grown lines within Uganda, as well as elites. The aim of the study was to identify potential volatile biochemical markers, if any, for the rice grain aroma trait. Certified rice seeds were obtained from the Uganda National Crops Resources Research Institute germplasm collection. The seeds were sown into experimental plots, under field conditions and the mature paddy harvested. Polished rice grains were heated to $80{ }^{\circ} \mathrm{C}$ and the liberated VOCs subjected to untargeted metabolite analysis using gas chromatography-time-of-flight mass spectrometry. In total, nine functional groups were present; hydrocarbons, alcohols, ketones, aldehydes, $\mathrm{N}$-containing compounds, S-containing compounds, esters, oxygen heterocycles and carboxylic acids. More specifically, 148 VOCs were identified across the 37 rice lines, of which 48 (32.4\%) including 2-acetyl-1-pyrroline (2-AP) appeared to elucidate the difference between non-aromatic and aromatic rice. Furthermore, $41(27.7 \%)$ VOCs were found to be significantly correlated with 2-AP abundance, the principle rice aroma compound. Amongst the $41 \mathrm{VOCs}$, only ten compounds were found to contribute highly towards variation in 2-AP abundance, indicative of their possible modulation roles in regard to rice aroma. Within the ten influential volatiles, three aroma active compounds; toluene, 1-hexanol, 2-ethyl and heptane, 2,2,4,6,6-pentamethyl- were established as the most reliable biochemical surrogates to the rice aroma trait. Thus, the aforementioned compounds may be used in rice breeding programme for enhancing development of the grain aroma trait.
\end{abstract}

Keywords: 2-acetyl-1-pyrroline, aroma, rice, volatile organic compounds

\section{Introduction}

Rice (Oryza sativa L.) is among the most important staple food crops for approximately two billion persons worldwide and a substantial source of income for several rural households (Sharma et al., 2018). Globally, several rice species exist including $O$. sativa thought to have originated in Asia (Kovach et al., 2009) and Oryza glaberrima of West African origin (Linares, 2002). The origin of a rice species is known to influence the physio-chemical attributes of the grain such as aroma and taste, primarily due to genetic inheritance (Rai et al., 2015). Considering aroma, the responsible gene betaine aldehyde dehydrogenase 2 (badh2) is believed to have different alleles (Bindusree et al., 2017), with specific allele configurations dependent upon species origin (Pachauri et al., 2010). Consequently, substantial variations in rice grain aroma has been observed in relation to differences in genetic backgrounds (Rai et al., 2015). 
Rice cultivars comprise non-aromatic and aromatic categories, of which aromatic cultivars exhibit a sweet popcorn flavour primarily attributed to 2-acetyl-1-pyrroline (2-AP) (Buttery et al., 1983). Aromatic rice is highly desirable amongst consumers, thus, commands high sensory preference and premium prices (Laizer et al., 2018). In spite the consensus that 2-AP contributes substantially towards rice aroma (Daygon et al., 2017), over 71 volatile compounds are thought to influence the perception of rice aroma (He et al., 2018). To date, more than 300 volatile compounds have been reported as constituting the multifaceted rice aroma trait (Champagne, 2008; Hashemi et al., 2013; Wakte et al., 2016; Hinge et al., 2019). Thus, rice aroma is most likely influenced by the comprehensive interactions of many different volatile compounds with relatively low odour thresholds (Hu et al., 2020). Recent evidence appears to support that 2-AP producing rice varieties may have different sensory properties (Daygon et al., 2016), implying that other volatile compounds contribute towards rice aroma or distinguish between non-aromatic and aromatic varieties (Hu et al., 2020). Therefore, earlier works by several authors intended to identify key volatile organic compounds responsible for the rice aroma are most likely unexhausted (Hu et al., 2020). For example, working with 14 Thai rice cultivars, Sansenya et al. (2018) identified 140 volatile organic compounds of which 18 key compounds exhibited substantial influence towards the rice aroma. The key volatiles were namely; hexanal, 1-pentanol, octanal, (E)-2-heptenal, 6-methyl-5-hepten-2-one, 1-hexanol, nonanal, 2-butoxy-ethanol, (E)-2-octenal, 1-tetradecene, 1-octen-3-ol, decanal, benzaldehyde, (E)-2-nonenal, 1-nonanol, benzyl alcohol, isovanillin and vanillin. In addition, the literature appears to suggest that substantial variations in the key rice aroma constituents is widespread and primarily dependent on genetic considerations (Ajarayasiri \& Chaiseri, 2008; Stefano \& Agronomia, 2011; Mahattanatawee \& Rouseff, 2014). Within genetic homologues, variations in rice aroma constituents is reportedly influenced by differences in cultivation environments, agro-cultural practices and post-harvest handling practices (Calingacion et al., 2015; Wakte et al., 2016; Xu et al., 2018). This state of affairs consequentially complicates efforts by national rice breeding programs in development of varieties with enhanced aroma traits.

With regard to Uganda, several rice germplasm were found to exhibit substantial variations in 2-AP abundance and most significantly, within similar aromatic categories (Ocan et al., 2019). The implication being that the substantial intra-group variations are most likely due to other VOCs, besides 2-AP, substantially contributing towards the rice aroma (Cho et al., 2013; Sansenya et al., 2018). However, the key volatiles thought to be responsible for the aromatic profile of commonly grown rice lines in Uganda are not well understood. Thus, the present study aimed to profile the volatile compounds in non-aromatic and aromatic rice lines commonly grown in Uganda to aid establishment of key volatile compounds as potential biochemical markers for the aroma trait.

\section{Method}

\subsection{Selection of Rice Germplasm}

Thirty seven commonly grown rice germplasm, 300 g each, were collected from the Rice Research Unit at the National Crops Resources Research Institute (NaCRRI), Uganda. The selected rice germplasm comprised of common and elite lines with different parental lineage and aroma classification (Table 1). 
Table 1. Geographic origin, genetic backgrounds (crosses, pedigree) and aroma classification of the 37 selected rice lines

\begin{tabular}{|c|c|c|c|c|}
\hline Lines & Crosses & Pedigree & Origin & Aroma classification \\
\hline AGRA 41 & $O$. sativa indica $\times O$. sativa indica & AGRA-CRI-UPL-3-4 & AfricaRice, Benin & Aromatic \\
\hline AGRA 55 & O. sativa indica $\times O$. sativa indica & AGRA-CRI-UPL-4-4 & CRI, Ghana & Aromatic \\
\hline AGRA 60 & $O$. sativa indica $\times O$. sativa indica & AGRA-CRI-UPL-4-13 & CRI, Ghana & Aromatic \\
\hline AGRA 78 & O. sativa indica $\times O$. sativa indica & AGRA-CRI-UPL-2-1 & CRI, Ghana & Aromatic \\
\hline ART 4 & O. sativa indica $\times O$. sativa indica & ART15-22-10-8-1-B-2-2 & AfricaRice, Nigeria & Aromatic \\
\hline ART 7 & O. sativa indica $\times O$. sativa indica & ART15-17-7-8-1-1-1-B-1-1 & AfricaRice, Nigeria & Aromatic \\
\hline ART 10 & O. sativa indica $\times O$. sativa indica & ART15-21-2-4-1-B-1-B-1-1 & AfricaRice, Nigeria & Aromatic \\
\hline ARU 1190 & O. sativa japonica $\times O$. glaberrima & IR77454-22-B-20-2-2-B-TGR2 & AfricaRice, Nigeria & Non-aromatic \\
\hline Basmati 370 & - & - & - & Aromatic \\
\hline E 20 & $\begin{array}{l}\text { O. sativa indica } \times \\
(\text { O. sativa } \times O . \text { glaberrima })\end{array}$ & IRAT325/WAB365-B-1H1-HB & NaCRRI, Uganda & Non-aromatic \\
\hline Kafaci & - & - & AfricaRice & Non-aromatic \\
\hline Komboka & O. sativa indica $\times O$. sativa indica & IR05N221 & IRRI, Philippines & Aromatic \\
\hline MET 3 & $O$. sativa indica $\times O$. barthi & ART35-114-1-6N-2 & AfricaRice, Nigeria & Aromatic \\
\hline MET 4 & O. sativa indica $\times O$. barthi & ART34-146-1-8N-1 & AfricaRice, Nigeria & Aromatic \\
\hline MET 6 & O. sativa indica $\times O$. barthi & ART35-49-1-4N-1 & AfricaRice, Nigeria & Aromatic \\
\hline MET 12 & O. sativa indica $\times O$. barthi & ART34-88-1-2-B-1 & AfricaRice, Nigeria & Aromatic \\
\hline MET 13 & O. sativa indica $\times O$. barthi & ART34-113-3-2-B-1 & AfricaRice, Nigeria & Aromatic \\
\hline MET 14 & O. sativa indica $\times O$. barthi & ART34-256-3-1-B-2 & AfricaRice, Nigeria & Aromatic \\
\hline MET 16 & $O$. sativa indica $\times O$. barthi & ART35-272-1-2-B-1 & AfricaRice, Nigeria & Aromatic \\
\hline MET 25 & O. barthi interspecific lines & ART27-58-6-2-1-1-3-2 & AfricaRice, Nigeria & Non-aromatic \\
\hline MET 28 & O. barthi interspecific lines & ART27-58-6-2-2-2 & AfricaRice, Nigeria & Non-aromatic \\
\hline MET 33 & O. sativa indica $\times O$. barthi & ART27-58-6-2-1-1-3-1 & AfricaRice, Nigeria & Non-aromatic \\
\hline MET 38 & O. barthi interspecific lines & ART16-5-9-22-3-B-B-2 & AfricaRice, Nigeria & Non-aromatic \\
\hline MET 40 & O. sativa indica $\times O$. barthi & ART27-190-1-4-2-1-1-3 & AfricaRice, Nigeria & Aromatic \\
\hline Namche 1 & - & - & NaCRRI, Uganda & Non-aromatic \\
\hline Namche 2 & $\begin{array}{l}\text { O. sativa japonica } \times \\
(\text { O. sativa japonica } \times \text { O. glaberrima })\end{array}$ & NM7-8-2-B-P-11-6 & NaCRRI, Uganda & Non-aromatic \\
\hline Namche 4 & $O$. sativa indica $\times O$. sativa indica & ART3-11L1P1-B-B-2 & AfricaRice, Nigeria & Non-aromatic \\
\hline Namche 5 & O. sativa indica $\times O$. sativa indica & NM7-27-1- B-P-77-6 & NaCRRI, Uganda & Non-aromatic \\
\hline Namche 6 & O. sativa indica $\times O$. sativa indica & NM7-5-2- B-P-79-7 & NaCRRI, Uganda & Non-aromatic \\
\hline Nerica 4 & O. sativa japonica $\times$ O. glaberrima & WAB 450-1-B-P-91-HB & AfricaRice, Côte d'Ivoire & Non-aromatic \\
\hline Nerica 10 & - & WAB 450-11-1-1-P41-HB & WARDA/Africa Rice & Aromatic \\
\hline Sande & O. sativa indica $\times O$. barthi & O. barthi interspecific lines & AfricaRice, Nigeria & Aromatic \\
\hline Supa 3 & O. sativa indica $\times O$. sativa indica & IR 97011-7-7-3-1-B & IRRI, Philippines & Aromatic \\
\hline Supa 5 & O. sativa indica $\times O$. sativa indica & - & IRRI, Philippines & Aromatic \\
\hline Supa 6 & O. sativa indica $\times O$. sativa indica & IR 9712-4-1-2-1-1 & IRRI, Philippines & Aromatic \\
\hline Supa 1052 & $O$. sativa indica $\times O$. sativa indica & - & AfricaRice, Nigeria & Aromatic \\
\hline Yasmin aromatic & O. sativa indica $\times O$. sativa japonica & - & Egypt & Aromatic \\
\hline
\end{tabular}

Source: Kanaabi et al. (2018).

\subsection{Preparation of the Polished Rice Grains}

Paddy rice lines were processed according to the method by He et al. (2018) with slight modifications. Rice lines (300 $\mathrm{g}$ each) were submerged in $1 \mathrm{~L}$ distilled water and the floated kernels immediately discarded. The settled rice kernels were briefly washed and then sun dried for $48 \mathrm{~h}$, final moisture content approximately $14 \%$. The dry paddy rice were milled and polished using a bench top milling machine (Satake, Tokyo, Japan). Fifty (50) grams of polished rice grains were vacuum packed into air-tight polyethylene bags and delivered to the School of Agriculture and Food Sciences laboratory, University of Queensland, Australia for biochemical analyses.

\subsection{Identification and Quantification of Volatile Organic Compounds}

Polished rice grains were ground using a cryogrinder (Qiagen, Hilden, Germany) to particle size $<25 \mu \mathrm{m}$, and the flours weighed $(1 \mathrm{~g})$ into autosampler tubes. The tightly sealed tubes, in triplicates, were stored at $-80{ }^{\circ} \mathrm{C}$ until required. Pre-analysis, the frozen flours were left overnight at room temperature to equilibrate (Daygon et al., 2017). Thawed flours were then randomised and analysed in batches of 50. Blank samples were run before analysis of the rice flours to equilibrate the machine and quality control (QC) standards were placed at every $10^{\text {th }}$ queue position. Rice flours were assayed following the method by Daygon et al. (2017). In summary, rice flours were 
heated to $80^{\circ} \mathrm{C}$ with agitation for $10 \mathrm{~min}$ on a CombiPal Autosampler (Agilent, CA, USA) to release the VOCs. Liberated VOCs within the tube headspace $(1.5 \mathrm{ml})$ were collected using $2.5 \mathrm{ml}$ headspace syringes at $80{ }^{\circ} \mathrm{C}$ and injected in splitless mode (Pegasus 4D GC $\times$ GC-TOF-MS Leco; St. Joseph, MI, USA). Temperature of the gas chromatographer (GC) inlet and transfer lines were maintained at $250{ }^{\circ} \mathrm{C}$. Separation was performed first on a primary column (Agilent DB-624UI midpolar, $30 \mathrm{~m} \times 250 \mu \mathrm{m} \times 1.4 \mu \mathrm{m}$; Agilent, CA, USA) and then on a secondary column, Stabilwax (polar, $0.9 \mathrm{~m} \times 250 \mu \mathrm{m} \times 0.50 \mu \mathrm{m}$; Restek, Bellefon, USA). The primary column was initially set to $45{ }^{\circ} \mathrm{C}$ for $1 \mathrm{~min}$ and then ramped at a rate of $10{ }^{\circ} \mathrm{C} / \mathrm{min}$ to $235^{\circ} \mathrm{C}$. The secondary column and the modulator were set at $15^{\circ} \mathrm{C}$ and $25^{\circ} \mathrm{C}$ higher than the primary column respectively, during the entire run. The modulation period was set at $2.5 \mathrm{~s}$, with $0.4 \mathrm{~s}$ hot pulse time and $0.85 \mathrm{~s}$ cool time between stages. The carrier gas (helium) was maintained at a constant flow rate of $1 \mathrm{ml} / \mathrm{min}$. Data were acquired using a TOF-MS after a $200 \mathrm{~s}$ delay with an acquisition rate of 200 spectra/s. The MS scanned analytes within the mass range of 35 to $500 \mathrm{~m} / \mathrm{z}$ and the ion source was held at $240{ }^{\circ} \mathrm{C}$.

Data pre-processing, alignment and noise correction were done using ChromaTof v4.50. Signal to noise ratio was set at 25. The absence of instrument drift and batch effects was verified using the QC standards and technical replicates. Identification of VOCs was achieved through comparison of retention time and electron ionization (EI) fragmentation patterns of rice flours to an in-house mass spectral library created by running authentic analytical standards (Daygon et al., 2017). The relative amounts of VOCs were calculated by measuring the area under the curve of the VOC peak.

\subsection{Data Analysis}

Overall mean abundance of VOCs, representative of previously established non-aromatic and aromatic rice lines (Ocan et al., 2019), were subjected to the Student's T-test at 5\% significance level using Genstat software $18^{\text {th }} \mathrm{Ed}$. (VSN International, Hemel Hempstead, UK). Correlation analysis between and within VOCs significantly ( $<<$ 0.05 ) associated to 2-AP abundance was conducted. Consequentially, VOCs significantly correlated with 2-AP abundance were subjected to simple regression analysis in a bid to determine the key volatiles, if any, as potential biochemical markers for the rice aroma trait.

\section{Results}

\subsection{Identification and Classification of the Rice Volatile Organic Compounds}

Aliquots of the volatile organic compounds collected from the 37 rice lines were individually subjected to GC $\times$ GC-TOF-MS analysis. In totality, 148 VOCs were identified and classified using ChemDraw software ver. 12.0 (PerkinElmer, Waltham, MA, USA) into their respective functional groups (Table 2). 
Table 2. Organic compounds detected in the volatile fraction of the 37 rice lines at $80{ }^{\circ} \mathrm{C}$ using gas chromatography-time-of-flight mass spectrometry

\begin{tabular}{|c|c|}
\hline Class & Volatile organic compounds (VOCs) \\
\hline 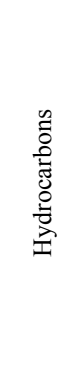 & $\begin{array}{l}\text { 1,3-Pentadiene, (Z)-; 10-Methylnonadecane; 1-Dodecene; Heptylcyclohexane; 1-Iodo-2-methylundecane; 2,3-Dioxabicyclo[2.2.1]heptane, } \\
\text { 1-methyl-; 3-Dodecene, (Z)-; 4-Tridecene, (Z)-; 7-Tetradecene; á-Phellandrene; a -Pinene; Benzene, 1,2,3-trimethyl-; Benzene, } \\
\text { 1,2,4,5-tetramethyl-; Benzene, 1,2,4-trimethyl-; Benzene, 1-ethyl-2-methyl-; Benzene, 1-ethyl-3-methyl-; Benzene, 1-ethyl-4-methyl-; } \\
\text { Benzene, 1-methyl-3-(1-methylethyl)-; Benzene, 2-ethyl-1,4-dimethyl-; Benzene, propyl-; Bicyclo[3.1.0]hex-2-ene, } \\
\text { 4-methyl-1-(1-methylethyl)-; Borine, ethyldispropyl-; Camphene; ç-Terpinene; Cyclohexane; Cyclohexane, hexyl-; Cyclohexene, } \\
\text { 1-methyl-4-(1-methylethylidene)-; Cyclopentane, methyl-; Decane, 2,3,5,8-tetramethyl-; Decane, 4-methyl-; Dodecane, 2,6,10-trimethyl-; } \\
\text { Ethylbenzene; Furan, 2-pentyl-; Heptadecane; Heptane, 2,2,4,6,6-pentamethyl-; Hexadecane; Hexane, 2,2,3-trimethyl-; Hexane, } \\
\text { 2,3-dimethyl-; Hexane, 2,4-dimethyl-; Hexane, 3-methyl-; Longifolene; Longipinane, (E)-; n-Hexane; Nonadecane; Octane, 4-ethyl-; } \\
\text { o-cymene; Pentane, 2,2,3-trimethyl-; Pentane; Propylene oxide; p-Xylene; Styrene; Syn-Tricyclo[5.1.0.0(2,4)]oct-5-ene, } \\
\text { 3,3,5,6,8,8-hexamethyl-; Tetradecane; Toluene; Tridecane, 3-methyl-; Undecane and Undecane, 2,6-dimethyl- }\end{array}$ \\
\hline $\begin{array}{l}\frac{0}{8} \\
\frac{1}{0} \\
\frac{0}{2}\end{array}$ & $\begin{array}{l}\text { 1-Butanol, 3-methyl-; 1-Butanol; 1-dodecanol, 3,7,11-trimethyl-; 1-Hexanol; 1-hexanol, 2-ethyl-; 1-Nonanol; 1-Octanol, 2-butyl-; } \\
\text { 1-Octen-3-ol; 1-Pentanol; 1-propanol; 1-Propanol, 2-methyl-; 2-Hexyl-1-octanol, (Z)-; 2-Pentanol; 2-Penten-1-ol, (Z)-; 2-Propanol, } \\
\text { 1-methoxy-; 2-Propanol, 2-methyl-; 3-Methyl-3-buten-1-ol; 4,6-Heptadien-1-ol, (Z)-; 4-Hexen-1-ol, (Z)-; Benzyl alcohol; Cyclohexanol; } \\
\text { Ethanol; Ethanol, 2-butoxy-; Isopropyl Alcohol and Z-10-Pentadecen-1-ol }\end{array}$ \\
\hline 离 & $\begin{array}{l}\text { 1-Penten-3-one, 2-methyl-; 2,3-Butanedione; 2,3-Octanedione; 2-Butanone; 2-Heptanone; 2-Hexanone; 2-Octanone; 2-Pentanone; } \\
\text { 3-Octen-2-one; 3-Octen-2-one, (E)-; 3-Pentanone, 2-methyl-; 5-Hepten-2-one, 6-methyl-; Acetoin and Methyl Isobutyl Ketone }\end{array}$ \\
\hline 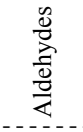 & $\begin{array}{l}\text { 2-Heptenal, (Z)-; 2-Nonenal, (E)-; 2-Octenal, (E)-; 2-Propanal, 2-methyl-; Benzaldehyde; Butanal, 2-methyl-; Butanal, 3-methyl-; Decanal; } \\
\text { Heptanal; Hexanal; Methyl acetoacetate; Nonanal; Octanal; Pentanal and Propanal, 2-methyl- }\end{array}$ \\
\hline 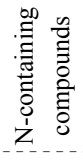 & $\begin{array}{l}\text { 2-Propanamine; 3-Butenamide; acetic acid, [(aminocarbonyl)amino]oxo-; acetic acid, cyno-; Ala-Gly; Furmaronitrile; Hydroxylamine, } \\
\text { O-decyl-; L-Ala-L-Ala-L-Ala; N,N Dimethyl-3,4-methylenedioxyamphetamine; N-à-(tert-Butoxycarbonyl)-L-Alanine; N-Acetylglycine } \\
\text { and Oxime, methoxy-phenyl- }\end{array}$ \\
\hline 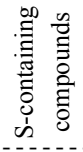 & $\begin{array}{l}\text { 2-Mercaptoethanol; 2-Undecanethiol, 2-methyl-; Bicyclo[3.1.1]heptane, 6,6-dimethyl-2-methylene-, (1S)-; Cyclohexene, } \\
\text { 1-methyl-4-(1-methylethenyl)-, (S)-; Dimethyl sulfide; Dimethyl trisulfides; Disulfide, dimethyl-; Methanesulfonic anhydride; } \\
\text { Methanethiol and Sulfur dioxide }\end{array}$ \\
\hline$\frac{\sqrt[0]{0}}{0}$ & $\begin{array}{l}\text {-2-propyl acetate; } 2 \text {-Propenoic acid, butyl ester; Dodecanoic acid, } 11 \text {-amino-, methyl ester; Hexanoic acid, } 2 \text {-propenyl ester and } \\
\text { acid dibutyl ester }\end{array}$ \\
\hline 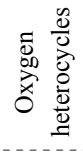 & $\begin{array}{l}\text { 2(3H)-Furanone,dihydro-3,5-dimethyl-; 2-Acetyl-1-pyrroline; 2-n-Butyl furan; 7-Oxabicyclo[4.1.0]heptane, 3-oxiranyl-; Creatinine; } \\
\text { Cyclopentanone, 2-methyl-; Furan, 2,5-dihydro-; Furan, 2-ethyl- and Oxepine, 2,7-dimethyl- }\end{array}$ \\
\hline 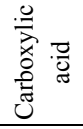 & Formic acid \\
\hline
\end{tabular}

With regard to relative proportion, hydrocarbons constituted the most abundant fraction, $31.1 \%$ (56 VOCs). In descending order; alcohols constituted, 16.9\% (25 VOCs), followed by ketones and aldehydes each at $9.5 \%$ (14 VOCs) then N-containing compounds, $8.8 \%$ (13 VOCs) and S-containing compounds, 6.6\% (10 VOCs). At the tail end, carboxylic acid comprised $0.7 \%$ (1 VOC), esters 4.1\% (6 VOCs) and O-heterocycles 6.1\% (9 VOCs) (Table 2).

\subsection{Overall Mean Abundance of the Key VOCs within Non-aromatic and Aromatic Rice Lines}

A comparison between the overall mean abundance of the 148 VOCs across non-aromatic and aromatic lines revealed 48 VOCs that appeared to differentiate the 37 rice lines into two aroma categories (Table 3 ). 
Table 3. Comparison between the overall means of non-aromatic and aromatic rice lines with regards to VOCs that registered significant $(\mathrm{p}<0.05)$ differences

\begin{tabular}{|c|c|c|c|c|}
\hline \multirow{2}{*}{ Volatile organic compounds (VOCs) } & \multicolumn{2}{|c|}{ Mass ion abundance } & \multirow{2}{*}{$p$-value } & \multirow{2}{*}{ Aroma description } \\
\hline & Non-aromatic & Aromatic & & \\
\hline \multicolumn{5}{|l|}{ Hydrocarbons } \\
\hline 10-Methylnonadecane & 240859 & 318805 & 0.023 & \\
\hline Benzene, 1,2,3-trimethyl- & 249123 & 378067 & 0.003 & \\
\hline Benzene, 1,2,4,5-tetramethyl- & 124163 & 204082 & 0.003 & \\
\hline Benzene, 1,2,4-trimethyl- & 43679 & 65154 & 0.039 & \\
\hline Benzene, 1-ethyl-2-methyl- & 202450 & 268955 & 0.060 & \\
\hline Benzene, 1-ethyl-3-methyl- & 83546 & 161805 & 0.002 & \\
\hline Benzene, 1-ethyl-4-methyl- & 46762 & 91274 & 0.006 & \\
\hline Benzene, 2-ethyl-1,4-dimethyl- & 40910 & 81014 & 0.002 & \\
\hline Decane, 4-methyl- & 524809 & 848046 & 0.002 & \\
\hline Heptane, 2,2,4,6,6-pentamethyl- & 442186 & 1012860 & 0.002 & \\
\hline Hexadecane & 168298 & 209576 & 0.001 & \\
\hline Hexane, 2,2,3-trimethyl- & 325421 & 489209 & 0.014 & \\
\hline Hexane, 2,3-dimethyl- & 16592 & 23325 & 0.026 & \\
\hline Hexane, 2,4-dimethyl- & 532162 & 641542 & 0.001 & \\
\hline Hexane, 3-methyl- & 565827 & 681090 & 0.033 & \\
\hline Longipinane, (E)- & 11278 & 21693 & 0.039 & \\
\hline $\mathrm{n}$-Hexane & 36349229 & 35497155 & 0.033 & \\
\hline Nonadecane & 416779 & 496701 & 0.011 & Sweet, rosy ${ }^{5}$ \\
\hline Octane, 4-ethyl- & 194228 & 326142 & $<0.001$ & \\
\hline o-cymene & 46860 & 101667 & 0.003 & \\
\hline Tetradecane & 2499182 & 3273103 & 0.001 & Gasoline-like, alkane ${ }^{5}$ \\
\hline Toluene & 3513759 & 4210663 & 0.006 & Sweet, pungent, ethereal ${ }^{5}$ \\
\hline Tridecane, 3-methyl- & 408801 & 468244 & 0.035 & \\
\hline Undecane, 2,6-dimethyl & 266999 & 389806 & 0.003 & \\
\hline \multicolumn{5}{|l|}{ Alcohols } \\
\hline 1-Hexanol, 2-ethyl- & 692538 & 924883 & 0.029 & Sweet, green odour ${ }^{1}$ \\
\hline 1-Octanol, 2-butyl- & 487391 & 620564 & 0.013 & \\
\hline 1-Octen-3-ol & 1485477 & 1897820 & 0.012 & Raw mushroom, straw ${ }^{5}$ \\
\hline 1-Pentanol & 1166224 & 1811653 & 0.009 & Plastic, green, fusel oil-like \\
\hline 2-Penten-1-ol, (Z)- & 566821 & 676221 & 0.018 & Sweet strong odour ${ }^{1}$ \\
\hline Cyclohexanol & 1235725 & 1911857 & 0.001 & Camphor-like $^{6}$ \\
\hline \multicolumn{5}{|l|}{ Ketones } \\
\hline 1-Penten-3-one, 2-methyl- & 123502 & 329569 & 0.002 & \\
\hline 2-Heptanone & 701518 & 1110088 & 0.011 & Fruit, spicy odour ${ }^{1}$ \\
\hline 2-Octanone & 94051 & 146259 & 0.016 & \\
\hline 5-Hepten-2-one, 6-methyl- & 63794 & 78788 & 0.041 & \\
\hline Cyclopentanone, 2-methyl- & 389186 & 564195 & 0.003 & \\
\hline \multicolumn{5}{|l|}{ Aldehydes } \\
\hline 2-Heptenal, (Z)- & 260866 & 307491 & 0.039 & Herbaceous, fatty, green ${ }^{5}$ \\
\hline Benzaldehyde & 4625220 & 5986177 & 0.001 & Nutty, sweet, bitter odour ${ }^{2,4}$ \\
\hline Heptanal & 497022 & 586418 & 0.045 & Fatty, rancid ${ }^{2}$ \\
\hline Pentanal & 2399867 & 3147404 & 0.018 & Woody, fruity odour ${ }^{2}$ \\
\hline \multicolumn{5}{|l|}{$N$-containing compounds } \\
\hline Acetic acid, cyano- & 1283377 & 1689341 & 0.038 & \\
\hline Hydroxylamine, O-decyl- & 191618 & 347120 & $<0.001$ & \\
\hline \multicolumn{5}{|l|}{ S-containing compound } \\
\hline Disulfide, dimethyl & 3285490 & 3640312 & 0.002 & \\
\hline \multicolumn{5}{|l|}{ Esters } \\
\hline Hexanoic acid, 2-propenyl ester & 74118 & 130270 & 0.022 & \\
\hline Methyl acetoacetate & 179467 & 291531 & $<0.001$ & \\
\hline \multicolumn{5}{|l|}{ O-heterocycles } \\
\hline 2-Acetyl-1-pyrroline & 92964 & 480846 & 0.002 & Sweet popcorn ${ }^{3}$ \\
\hline 2,3-Dioxabicyclo[2.2.1]heptane, 1-methyl- & 19206 & 54043 & $<0.001$ & \\
\hline 7-Oxabicyclo[4.1.0]heptane, 3-oxiranyl- & 58948 & 97305 & 0.04 & \\
\hline Furan, 2-pentyl- & 2019058 & 2337019 & 0.043 & $\begin{array}{l}\text { Floral, fruity, nutty, almond, } \\
\text { beany, green, buttery }\end{array}$ \\
\hline
\end{tabular}

Note. ${ }^{1}=$ Wilkie et al. (2007); ${ }^{2}=$ Givianrad (2012); ${ }^{3}=$ Buttery et al. $(1983) ;{ }^{4}=$ He et al. (2018); ${ }^{5}=$ Hinge et al. (2016); ${ }^{6}=$ Xia et al. (2017). 
Considering the 48 VOCs that revealed significantly $(\mathrm{p}<0.05)$ different quantities between non-aromatic and aromatic rice categories; hydrocarbons constituted the majority at $50.0 \%$ (24 VOCs), followed by alcohols $12.5 \%$ (6 VOCs), ketones $10.4 \%$ (5 VOCs), aldehydes $8.3 \%$ (4 VOCs), O-heterocycles $8.3 \%$ (4 VOCs), Ncontaining compounds $4.2 \%$ ( 2 VOCs), esters $4.2 \%$ ( 2 VOCs) and S-containing compounds $2.1 \%$ (1 VOC) (Table 3). With the exception of the carboxylic acid group, the total number of functional groups present within both the non-aromatic and aromatic rice categories remained unchanged.

However, a substantial reduction in the number of VOCs established as possible discriminators between the rice aroma categories (Table 3) and the total number of VOCs that constitute rice aroma (Table 2) was observed. Amongst the 148 VOCs identified as constituting rice aroma, only 48 compounds seem to discriminate between non-aromatic and aromatic rice lines, a $67.6 \%$ reduction. Specifically, the percentage decrease within the respective functional groups were dissimilar, i.e., O-heterocycles (55.6\% reduction), had the lowest decrease followed by hydrocarbons (57.1\% reduction), ketones (64.3\% reduction), Esters (60\% reduction), aldehydes (73.3\% reduction), alcohols (76.9\% reduction), N-containing compounds (83.3\% reduction) and S-containing compounds ( $90 \%$ reduction). Out of the nine functional groups earlier determined (Table 2), only five namely; hydrocarbons, alcohols, ketones, aldehydes and O-heterocycles, had compounds with specific rice aroma descriptions (Table 3). For all 15 VOCs with known rice aroma descriptions, the non-aromatic category registered significantly $(\mathrm{p}<0.05)$ lower abundance compared to the aromatic. Intriguingly, the observation was also true for compounds with undesirable aroma descriptions, i.e., tetradecane (gasoline-like), 1-pentanol (plastic, fusel oil-like) and heptanal (rancid).

\subsection{Relationships, between and within, 2-Acetyl-1-pyrroline and Associated Volatile Organic Compounds}

A correlation analysis of the 148 VOCs (Table 2) revealed 12 (8.1\%) compounds to be highly associated to 2-AP, while $13(8.7 \%)$ compounds were moderately associated and $16(10.8 \%)$ compounds weakly associated (Tables $4 \mathrm{a}, 4 \mathrm{~b}$ and $4 \mathrm{c}$ ). Among the highly associated compounds to 2-AP, five functional groups were represented (Table $4 a$ ), while for the moderately associated compounds three functional groups were present (Table $4 \mathrm{~b}$ ). On the other hand, several VOCs were determined as weakly associated to 2-AP (Table 4c), thus, of limited importance in the present study.

Furthermore, with the exception of methyl acetoacetate and undecane, 2,6-dimethyl-, all compounds found to be highly associated to 2-AP were also highly or moderately associated amongst themselves (Table 4a). For moderately associated compounds to 2-AP, it was noted that all compounds were either highly or moderately associated amongst each other with the exception of: cyclopentane, methyl; cyclohexane; 1-penten-3-one, 2-methyl and hexane, 2,3-dimethyl (Table 4b).

Comparison of the compounds in Table $4 \mathrm{a}$ to Table 3 revealed that 9 out of $12(91.7 \%)$ VOCs were common to both analyses. In addition, a similar comparison between compounds in Table $4 \mathrm{~b}$ to Table 3 revealed that 12 out of $13(92.3 \%)$ VOCs were common in both sets of results. Expectedly, out of the 16 VOCs that were determined as weakly associated to 2-AP, only five compounds namely; benzaldehyde; 7-Oxabicyclo[4.1.0]heptane, 3-oxiranyl-; 1-Octanol, 2-butyl-; tridecane, 3-methyl- and nonedecane were common to both sets of results (Tables $4 \mathrm{c}$ and 3). 
Table 4a. Correlation coefficient, between and within, 2-Acetyl-1-pyrroline and other highly associated volatile organic compounds

\begin{tabular}{|c|c|c|c|c|c|c|c|c|c|c|c|c|c|}
\hline & 2-AP & BZTM2 & BZTM3 & EDE & ETL & HAD & HNLE & HРTM & HPTM3 & MA & MID & Toluene & UDM \\
\hline 2-AP & - & & & & & & & & & & & & \\
\hline BZTM2 & $0.52^{* * *}$ & - & & & & & & & & & & & \\
\hline BZTM3 & $0.55^{* * *}$ & $0.87^{* * *}$ & - & & & & & & & & & & \\
\hline EDE & $0.53^{* * *}$ & $0.72 * * *$ & $0.82^{* * *}$ & - & & & & & & & & & \\
\hline ETL & $0.58^{* * *}$ & $0.59 * * *$ & $0.46^{* *}$ & $0.47 * *$ & - & & & & & & & & \\
\hline HAD & $0.56^{* * *}$ & $0.84 * * *$ & $0.91^{* * *}$ & $0.87 * * *$ & $0.43 * *$ & - & & & & & & & \\
\hline HNLE & $0.56^{* * *}$ & $0.61^{* * *}$ & $0.78^{* * *}$ & $0.75^{* * *}$ & $0.48^{* * *}$ & $0.73^{* * *}$ & - & & & & & & \\
\hline HРTM & $0.52 * * *$ & $0.93 * * *$ & $0.83^{* * *}$ & $0.64 * * *$ & $0.53 * * *$ & $0.84^{* * *}$ & $0.49^{* *}$ & - & & & & & \\
\hline HPTM3 & $0.59^{* * *}$ & $0.83 * * *$ & $0.79 * * *$ & $0.72 * * *$ & $0.68^{* * *}$ & $0.79^{* * *}$ & $0.64^{* * *}$ & $0.86^{* * *}$ & - & & & & \\
\hline MA & $0.52^{* * *}$ & 0.27 & 3.40 & $0.34^{*}$ & 0.08 & $0.37 *$ & 0.28 & 0.28 & 0.20 & - & & & \\
\hline MID & $0.52 * * *$ & $0.75^{* * *}$ & $0.83^{* * *}$ & $0.94 * * *$ & $0.52 * * *$ & $0.87 * * *$ & $0.76^{* * *}$ & $0.68 * * *$ & $0.78^{* * *}$ & $0.35^{*}$ & - & & \\
\hline Toluene & $0.63^{* * *}$ & $0.78 * * *$ & $0.70^{* * *}$ & $0.69 * * *$ & $0.59 * * *$ & $0.71^{* * *}$ & $0.58^{* * *}$ & $0.75^{* * *}$ & $0.73^{* * *}$ & $0.44 *$ & $0.66^{* * *}$ & - & \\
\hline UDM & $0.55^{* * *}$ & $0.77^{* * *}$ & $0.87 * * *$ & $0.90^{* * *}$ & $0.43^{*}$ & $0.94 * * *$ & $0.74 * * *$ & $0.74 * * *$ & $0.70^{* * *}$ & $0.39 *$ & $0.89^{* * *}$ & $0.65^{* * *}$ & - \\
\hline
\end{tabular}

Note. 2-AP = 2-Acetyl-1-pyrroline; BZTM2 = Benzene, 1,2,3-trimethyl-; ZTM3 = Benzene, 1,2,4,5-tetramethyl-; $\mathrm{EDE}=$ Hexadecane; ETL $=$ Ethanol, 2-butoxy; HAD = Hydroxylamine, O-decyl-; HPTM = Heptane, 2,2,4,6,6-pentamethyl-; HPTM3 = 2,3-Dioxabicyclo[2.2.1]heptane, 1-methyl-; MA = Methyl acetoacetate; UDM = Undecane, 2,6-dimethyl-; HNLE = 1-Hexanol, 2-ethyl- and MID = 10-Methylnonadecane.

$*=$ significant $(\mathrm{p}<0.05) ; * *=$ significant $(\mathrm{p}<0.01) ; * * *=\operatorname{significant}(\mathrm{p}<0.001)$.

Table 4b. Correlation coefficient, between and within, 2-Acetyl-1-pyrroline and other moderately associated volatile organic compounds

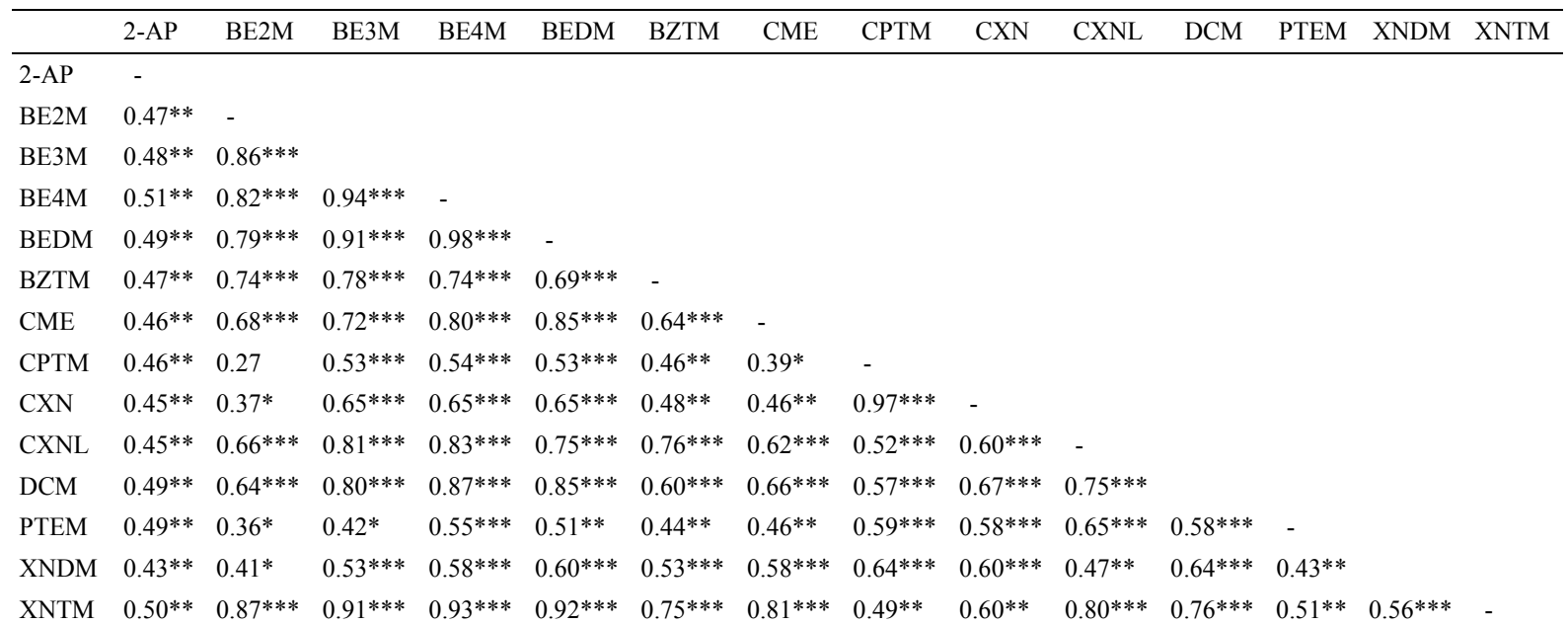

Note. $\mathrm{BZTM}=$ Benzene, 1,2,4-trimethyl-; BE2M = Benzene, 1-ethyl-2-methyl-; BE3M = Benzene, 1-ethyl-3-methyl-; BE4M = Benzene, 1-ethyl-4-methyl-; BEDM = Benzene, 2-ethyl-1,4-dimethyl-; CME = o-cymene; $\mathrm{CPTM}=$ Cyclopentane, methyl-; $\mathrm{CXN}=$ Cyclohexane; $\mathrm{CXNL}=$ Cyclohexanol; $\mathrm{DCM}=$ Decane, 4-methyl-; PTEM = 1-penten-3-one, 2-methyl-; XNDM = Hexane, 2,3-dimethyl- and XNTM = Hexane, 2,2,3-trimethyl-.

$*=$ significant $(\mathrm{p}<0.05) ; * *=$ significant $(\mathrm{p}<0.01) ; * * *=\operatorname{significant}(\mathrm{p}<0.001)$. 
Table 4c. Correlation coefficient, between and within, 2-Acetyl-1-pyrroline and other weakly associated volatile organic compounds

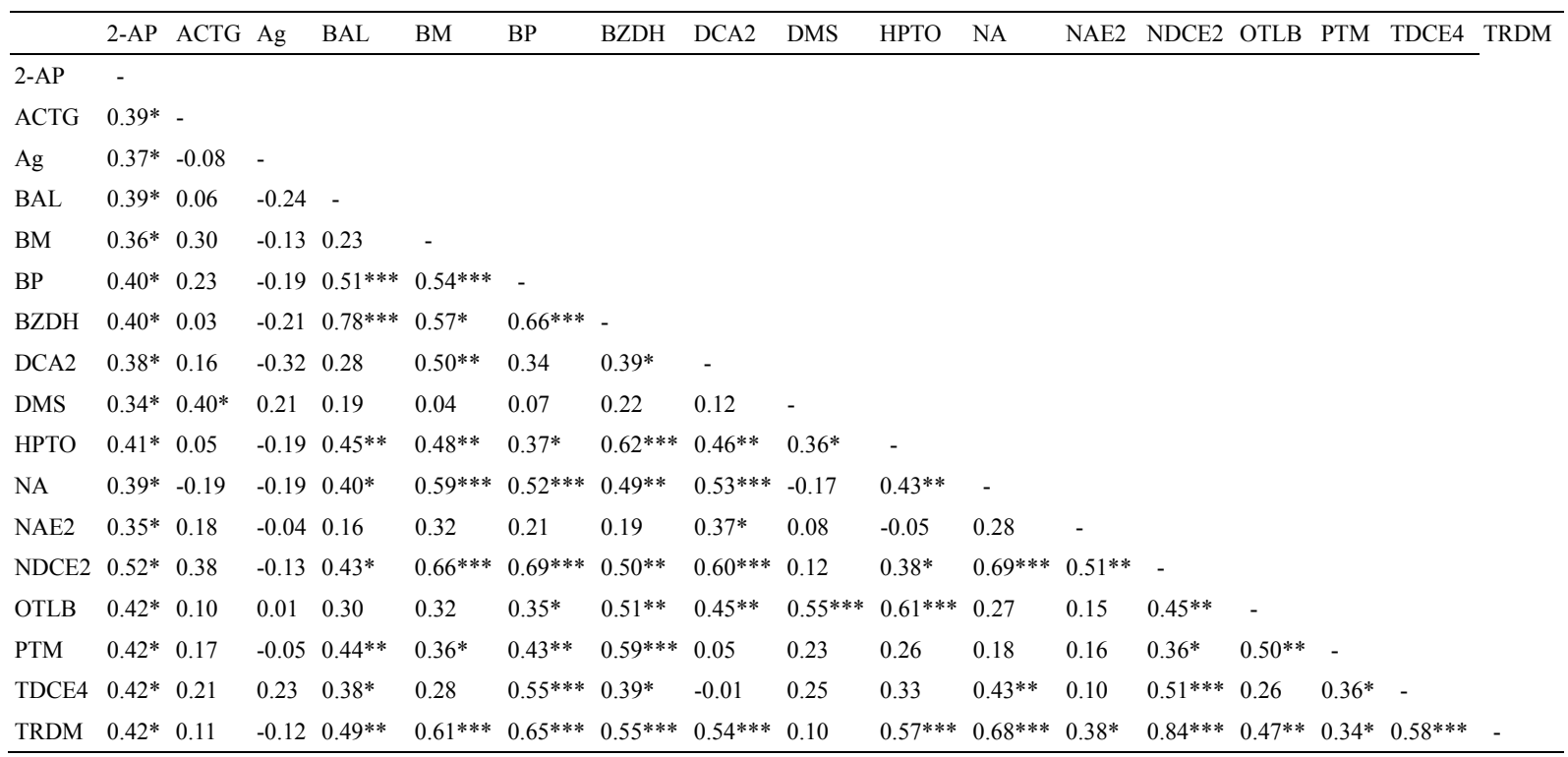

Note. ACTG = N-Acetylglycine; $\mathrm{Ag}=$ Ala-Gly; $\mathrm{BAL}=$ Benzyl alcohol; $\mathrm{BM}=$ Butanal, 2-methyl-; $\mathrm{BP}=$ Benzene, propyl; $\mathrm{BZDH}=$ Benzaldehyde; $\mathrm{DCA} 2=$ Decanal; $\mathrm{DMS}=$ Dimethyl sulfide; $\mathrm{HPTO}=$ 7-Oxabicyclo[4.1.0]heptane, 3-oxiranyl-; NA = Nonanal; $\quad$ NAE2 $=$ 2-Nonenal; NDCE2 $=$ Nonadecane; OTLB = 1-Octanol, 2-butyl-; PTM = Pentane, 2,2,3-trimethyl-; TDCE4 $=4$-Tridecene, $(Z)$ and TRDM $=$ Tridecane, 3-methyl-.

$*=$ significant $(\mathrm{p}<0.05) ; * *=$ significant $(\mathrm{p}<0.01) ; * * *=\operatorname{significant}(\mathrm{p}<0.001)$.

\subsection{Relationships between the Key VOCs and 2-Acetyl-1-pyrroline Abundance}

Given the limitations of correlation in revealing cause-effect relationships, a regression analysis was further performed on all 41 VOCs earlier found to be associated to 2-AP (Tables $4 \mathrm{a}, 4 \mathrm{~b}$ and $4 \mathrm{c}$ ).

Table 5. Volatile organic compounds responsible for major variation in 2-AP abundance

\begin{tabular}{llllll}
\hline No. & Volatile organic compounds (VOCs) & Parameter & Standard error & $p$-value & $\mathrm{R}^{2}$ \\
\hline 1 & Toluene & 0.224 & 0.046 & $<0.001$ & 38.400 \\
2 & 2,3-Dioxabicyclo[2.2.1]heptane, 1-methyl- & 4.340 & 1.060 & $<0.001$ & 30.400 \\
3 & Hydroxylamine, O-decyl- & 1.414 & 0.366 & $<0.001$ & 27.900 \\
4 & Ethanol, 2-butoxy & 1.547 & 0.392 & $<0.001$ & 28.200 \\
5 & Undecane, 2,6-dimethyl- & 1.587 & 0.417 & $<0.001$ & 27.300 \\
6 & Methyl acetoacetate & 1.427 & 0.383 & $<0.001$ & 26.400 \\
7 & 1-Hexanol, 2-ethyl- & 0.782 & 0.210 & $<0.001$ & 26.200 \\
8 & Benzene, 1,2,3-trimethyl- & 1.383 & 0.382 & $<0.001$ & 25.200 \\
9 & Heptane, 2,2,4,6,6-pentamethyl- & 0.263 & 0.073 & $<0.001$ & 25.200 \\
10 & Benzene, 1,2,4,5-tetramethyl- & 2.253 & 0.635 & $<0.001$ & 24.300 \\
11 & Benzene, 1-ethyl-3-methyl- & 2.210 & 0.644 & 0.002 & 23.100 \\
12 & Nonadecane & 1.653 & 0.484 & 0.002 & 22.900 \\
13 & Hexadecane & 3.970 & 1.180 & 0.002 & 22.400 \\
14 & Hexane, 2,2,3-trimethyl- & 0.951 & 0.284 & 0.002 & 22.100 \\
15 & 10-Methylnonadecane & 2.095 & 0.628 & 0.002 & 22.000 \\
16 & Hexane, 2,3-dimethyl- & 5.000 & 0.628 & 0.007 & 22.000 \\
17 & 1-Penten-3-one, 2-methyl- & 0.557 & 0.171 & 0.002 & 21.100 \\
18 & Decane, 4-methyl- & 0.453 & 0.140 & 0.003 & 20.900 \\
19 & Benzene, 1-ethyl-4-methyl- & 3.350 & 1.040 & 0.003 & 20.800 \\
20 & Benzene, 2-ethyl-1,4-dimethyl- & 4.080 & 1.260 & 0.003 & 20.800 \\
21 & Benzene, 1-ethyl-2-methyl- & 2.177 & 0.688 & 0.003 & 20.000 \\
\hline
\end{tabular}


Regression analysis revealed two broad categories of compounds namely; highly $(\mathrm{p}<0.001)$ and moderately $(p>0.001 \leq 0.007)$ related to the variation in 2 -AP abundance (Table 5). In the highly related category, ten compounds were present and comprised five functional groups, i.e., hydrocarbons, alcohols, N-containing compounds, esters and O-heterocycles. Concerning the relative percentage abundance of functional groups within the highly related category, hydrocarbons constituted $50 \%$ followed by alcohols at $20 \%$ while Ncontaining compounds, esters and O-heterocycles constituted $10 \%$, each. Relatedly, in descending order of $\mathrm{R}^{2}$, VOCs under the highly related category were: toluene; 2,3-dioxabicyclo[2.2.1]heptane, 1-methyl-; hydroxylamine, O-decyl-; ethanol, 2-butoxy; undecane, 2,6-dimethyl-; methyl acetoacetate; 1-hexanol, 2-ethyl-; benzene, 1,2,3-trimethyl-; heptane, 2,2,4,6,6-pentamethyl- and benzene, 1,2,4,5-tetramethyl-. On the other hand, for the moderately related category, eleven compounds were present and two functional groups represented, i.e., hydrocarbons and ketones.

\section{Discussion}

\subsection{Identification and Classification of the Volatile Organic Compounds}

Earlier studies have revealed wide variations in the number and chemical nature of VOCs associated with rice grain aroma. For example; 47 volatiles in Lee et al. (2019), 50 volatiles in Shanthine et al. (2019) and 140 compounds in Sansenya et al. (2018). Thus, the number of 148 VOCs in the present study is more closely aligned to 140 VOCs as reported by Sansenya et al. (2018). The wide variation (47 148 VOCs) in-between the numbers of volatile organic compounds reported under the different studies appears most likely due to differences in the technologies employed, nature and number of cultivars utilized. Generally, studies involving more advance techniques such as GC $\times$ GC-TOF-MS and numerically more rice lines, appeared to report higher numbers of VOCs (Lee et al., 2019; Fatemi et al., 2014; Shanthine et al., 2019; Sansenya et al., 2018). In regards to functional groups, earlier studies revealed slight variations in the nature and number constituting rice aroma. In the present study, nine functional groups were established (Table 2). This number is in agreement with earlier works by Fatemi et al. (2014) and Lee et al. (2019). These authors, reported the rice aroma metabolites to comprise; ketones, aldehydes, alcohols, carboxylic acids, esters, hydrocarbons, oxygen heterocycles, $\mathrm{N}$-containing compounds, benzene and benzene derivatives. Considering the nature of the functional groups involved in rice aroma, the three studies were similar with the exception of S-containing compounds.

\subsection{Volatile Organic Compounds Able to Differentiate Non-aromatic and Aromatic Rice Categories}

The presence of several VOCs capable of differentiating between non-aromatic and aromatic rice lines is probably due to the genetic diversity of rice lines within Uganda (Table 3). Similar to earlier report by Hinge et al. (2019), most volatiles in both the rice aroma categories were similar, but with different propositions. Therefore, for the 15 VOCs with aroma description, aromatic rice lines on average had more abundance of the compounds. Intriguingly, these included three volatiles with undesirable aroma tints namely; tetradecane (gasoline-like), 1-pentanol (plastic, fusel oil-like) and heptanal (rancid). Among these compounds, the contribution of tetradecane is most likely negligible due to the high odour threshold limitation of most hydrocarbons (Hu et al., 2020). In contrast to Setyaningsih et al. (2019) who found; pentanal, hexanal, 2-pentyl-furan, 2,4-nonadienal, pyridine, 1-octen-3-ol and (E)-2-octenal as volatile markers capable of differentiating between non-aromatic and aromatic rice, the present study found no similarity. Given the similarities in methodology, the absence of commonality in the results was most likely due to genetic differences in the rice lines. This highlights the fact that identified volatile biochemical markers may only be applicable in differentiating between specific rice lines and within similar environs. The present study also revealed that not all key volatile biochemical compounds possess aroma descriptions. This could possibly be construed to imply that not all volatile biochemical markers are aroma active compounds. This would be in contrast to the study by Setyaningsih et al. (2019) in which all the seven volatile biochemical markers were aroma active compounds. Hence, the present study deemed it necessary to further refine the list of possible volatile biochemical markers by subjecting the VOCs to correlation and regression analyses.

\subsection{Relationships, between and within, 2-Acetyl-1-Pyrroline and Associated Volatile Organic Compounds}

Hinge et al. (2016) reported that 2-AP abundance was not correlated to other aroma active compounds in aromatic rice. The authors postulated that the expression pattern of 2-AP was probably unique and specifically delinked from other volatiles of aroma importance. However, recently Daygon et al. (2017) documented a strong correlation between 2-AP abundance and amine heterocycles, namely; 6-methyl, 5-oxo-2,3,4,5-tetrahydropyridine (6M5OTP), 2-acetylpyrrole, pyrrole and 1-pyrroline. In the present study, a total of 25 VOCs were found to be highly or moderately associated to 2-AP abundance (Tables $4 \mathrm{a}$ and $4 \mathrm{~b}$ ). These 25 compounds comprised of six functional groups; hydrocarbons, alcohols, ketones, N-containing compounds, 
esters and O-heterocycles. In contrast, the functional groups for the present study did not contain amine heterocycles as earlier reported by Daygon et al. (2017). This is probably due to genetic differences in the constitution of the rice lines studied. Specifically, the present study employed genotypes developed from $O$. barthi, O. longistaminata, O.glaberrima and O. sativa backgrounds (Kitara et al., 2015; Lamo et al., 2017; Kanaabi et al., 2018) in contrast to the O. sativa intra-specific crosses used by Daygon et al. (2017).

In the present study, hydrocarbons and alcohols constituted the highest percentage of VOCs correlated to 2-AP, cumulatively $84 \%$. In contrast, ketones, N-containing compounds, esters and O-heterocycles constituted only $16 \%$. This is consistent with the importance of alcohols and hydrocarbons in rice aroma as earlier reported by Stefano and Agronomia (2011) and Lee et al. (2019). The absence of carboxylic acids, S-containing compounds and aldehydes (Table 4a and 4b) may be construed to imply that these compounds are of limited contribution towards 2-AP synthesis. However, this would be in contradiction to the finding by earlier authors concerning the importance of S-containing compounds and aldehydes in rice aroma (Sansenya et al., 2018). Thus, it is important to note that the absence of correlation between a given volatile compound and 2-AP may not indicate limitations in its sensory contribution towards rice aroma. Interestingly, with the exception of; methyl acetoacetate, heptane, 2,2,4,6,6-pentamethyl- and ethanol, 2-butoxy, all compounds highly associated to 2-AP were equally highly associated to each other (Table 4a). This implies that methyl acetoacetate, heptane, 2,2,4,6,6-pentamethyl- and ethanol, 2-butoxy may be important through associated pathways such as those influencing antagonistic effects beneficial to the perception of rice grain aroma (Champagne et al., 2008; Chambers et al., 2013).

\subsection{Possible Volatile Biochemical Markers for the Rice Aroma Trait}

With regards to identifying possible volatile biochemical markers for the aroma trait in rice, earlier studies have established the reliability of GC-MS techniques coupled with multivariate analyses. Setyaningsih et al. (2019) reported that out of 51 volatile organic compounds subjected to principal component analysis, seven key-marker volatile compounds namely; pentanal, hexanal, 2-pentyl-furan, 2,4-nonadienal, pyridine, 1-octen-3-ol and (E)-2-octenal were able to differentiate between non-aromatic and aromatic rice. The authors postulated that these volatile compounds were key-markers for the rice aroma trait. Griglione et al. (2015) conducted rice aroma fingerprinting and consequentially identified three volatile biochemical markers; heptanal, octanal and 2-ethyl hexanol. In the present study, 21 volatile organic compounds were indicative of 2-AP abundance and differentiated between the non-aromatic and aromatic rice lines. Further multivariate analyses revealed; toluene; 2,3-dioxabicyclo[2.2.1] heptane, 1-methyl-; hydroxylamine, O-decyl-; ethanol, 2-butoxy; undecane, 2,6-dimethyl; methyl acetoacetate; 1-hexanol, 2-ethyl; benzene, 1,2,3-trimethyl-; heptane, 2,2,4,6,6-pentamethyl- and benzene, $1,2,4,5$-tetramethyl- as candidates for volatile biochemical markers of the rice aroma trait. With the exception of 2-ethyl hexanol, all identified volatile markers in the three studies were not similar (Griglione et al., 2015; Setyaningsih et al., 2019). Importantly, careful examination of all earlier reported volatile markers indicated that the compounds were aroma active. Thus, toluene, 1-hexanol, 2-ethyl and heptane, 2,2,4,6,6-pentamethyl- are potential volatile biochemical markers for the rice aroma trait.

In conclusion, volatile organic compounds associated with rice grain aroma are quantitatively enormous and of diverse functional group classification. However, it is apparent that not all VOCs associated to rice aroma aid in the segregation of the rice grains into non-aromatic and aromatic types as perceived by consumers. Further, amongst the VOCs associated with the aroma trait in rice grains, not all compounds contribute equally. A few VOCs are responsible for both the discrimination of rice into non-aromatic and aromatic groups in addition to aiding desired sensory perception. These three compounds, namely: toluene, 1-hexanol, 2-ethyl and heptane, 2,2,4,6,6-pentamethyl- are therefore potential volatile biomarkers for the rice aroma trait. Thus, the aforementioned compounds may be used in rice breeding programmes for enhancing development of the aroma trait.

\section{Acknowledgements}

This work was supported by the scientific partnership between Makerere University - Swedish International Development Cooperation Agency (SIDA) programme and the University of Queensland, Australia. Certified rice germplasm were donated by the Cereal Programme, National Crops Resources Research Institute (NaCRRI), Uganda.

\section{References}

Ajarayasiri, J., \& Chaiseri, S. (2008). Comparative Study on Aroma-Active Compounds in Thai, Black and White Glutinous Rice Varieties. Kasetsart Journal of Natural Sciences, 42, 715-722 
Bindusree, G., Natarajan, P., Kalva, S., \& Madasamy, P. (2017). Whole genome sequencing of Oryza sativa L. cv. Seeragasamba identifies a new fragrance allele in rice. PLOS ONE, 1-9. https://doi.org/10.1371/journal. pone.0188920

Buttery, R. G., Ling, L. C., Juliano, B. O., \& Jean, G. T. J. (1983). Cooked Rice Aroma and 2-Acetyl-1-pyrroline. Journal of Agricultural and Food Chemistry, 31(4), 821-826. https://doi.org/10.1021/jf00118a036

Calingacion, M., Fang, L., Quiatchon-Baeza, L., Mumm, R., Riedel, A., Hall, R. D., \& Fitzgerald, M. A. (2015). Delving deeper into technological innovations to understand differences in rice quality. Rice, 8(6), 1-10. https://doi.org/10.1186/s12284-015-0043-8

Chambers, E., \& Koppel, K. (2013). Associations of Volatile Compounds with Sensory Aroma and Flavor: The Complex Nature of Flavor. Molecules, 18, 4887-4905. https://doi.org/10.3390/molecules 18054887

Champagne, E. T. (2008). Rice Aroma and Flavor: A Literature Review. Cereal Chemistry, 85(4), $445-454$. https://doi.org/10.1094/CCHEM-85-4-0445

Cho, S., Nuijten, E., Shewfelt, R. L., \& Kaysa, S. J. (2013). Aroma chemistry of African Oryza glaberrima and Oryza sativa rice and their interspecific hybrids. Journal of Science of Food and Agriculture, 1-9. https://doi.org/10.1002/jsfa.6329

Daygon, V. D., Calingacion, M., Forster, L. C., Voss, J. J., De Schwartz, B. D., Ovenden, B., ... Fitzgerald, M. A. (2017). Metabolomics and genomics combine to unravel the pathway for the presence of fragrance in rice. Scientific Reports, 1-12. https://doi.org/10.1038/s41598-017-07693-9

Daygon, V. D., Prakash, S., Calingacion, M., Riedel, A., Ovenden, B., Snell, P., Mitchell, J., \& Fitzgerald, M. (2016). Understanding the Jasmine phenotype of rice through metabolite profiling and sensory evaluation. Metabolomics, 12(4), 1-15. https://doi.org/10.1007/s11306-016-0989-6

Fatemi, M. H., Malekzadeh, H., Ghassempour, A., \& Mahdavi, V. (2014). Analysis of flavor volatiles of some Iranian rice cultivars by SPME-GC-MS. Caspian Journal of Chemistry, 3, 35-43.

Givianrad, M. H. (2012). Characterization and Assessment of Flavor Compounds and Some Allergens in Three Iranian Rice Cultivars during Gelatinization Process by HS-SPME/GC-MS. Electronic Journal of Chemistry, 9(2), 716-728. https://doi.org/10.1016/j.foodchem.2014.09.082

Griglione, A., Liberto, E., Cordero, C., Bressanello, D., Cagliero, C., Rubiolo, P., ... Sgorbini, B. (2015). High-quality Italian rice cultivars: Chemical indices of ageing and aroma quality. Food Chemistry, 172, 305-313. https://doi.org/10.1016/j.foodchem.2014.09.082

Hashemi, F. S. G., Rafii, M. Y., Ismail, M. R., Mahmud, T. M. M., Rahim, H. A., Asfaliza, R., ... Latif, M. A. (2013). Biochemical, Genetic and Molecular Advances of Fragrance Characteristics in Rice. Critical Reviews in Plant Sciences, 32(6), 445-457. https://doi.org/10.1080/07352689.2013.807716

He, F., Qian, Y., Zhang, Y., Zhang, M., \& Qian, M. C. (2018). Aroma compounds generation in brown and polished rice during extrusion. Flavour Science, 103-106. https://doi.org/10.3217/978-3-85125-593-5-21

Hinge, V. R., Patil, H. B., \& Nadaf, A. B. (2016). Aroma volatile analyses and 2-AP characterization at various developmental stages in Basmati and Non-Basmati scented rice (Oryza sativa L.) cultivars. Rice, 9(38), 1-22. https://doi.org/10.1186/s12284-016-0113-6

Hinge, V., Zanan, R., Rashmi, D., \& Nadaf, A. (2019). Aroma Volatiles as Biomarkers for Characterizing Rice (Oryza sativa L.) Flavor Types and Their Biosynthesis. Science and Technology of Aroma, Flavor, and Fragrance in Rice (pp. 217-288). Apple Academic Press.

$\mathrm{Hu}$, X., Lu, L., Guo, Z., \& Zhu, Z. (2020). Volatile compounds, affecting factors and evaluation methods for rice aroma: A review. Trends in Food Science \& Technology, 97, 136-146. https://doi.org/10.1016/j.tifs.2020. 01.003

Kanaabi, M., Tusiime, G., Tukamuhabwa, P., Andaku, J., Ocan, D., \& Jimmy, L. (2018). Evaluation of Rice Germplasm Reveals Sources of Bacterial Leaf Streak Disease Resistance in Uganda. Journal of Food Security, 6(4), 163-169. https://doi.org/10.12691/jfs-6-4-4

Kitara, I. O., Lamo, J., Edema, R., Gipson, P., \& Rubaihayo P. R. (2015). Amylose content and grain appearance traits in rice genotypes. African Crop Science Journal, 27(3), 501-513. https://doi.org/10.4314/acsj.v27i3.12

Kovach, M. J., Calingacion, M. N., Fitzgerald, M. A., \& Mccouch, S. R. (2009). The origin and evolution of fragrance in rice (Oryza sativa L.). PNAS, 106(34), 14444-14449. https://doi.org/10.1073_pnas.0904077106 
Laizer, J. S., Baharanyi, N. R., Tackie, D. N. O., Zabawa, R., \& Kadigi, R. M. (2018). Determinants of Consumer Preference for and Expenditure on Rice in the Kilimanjaro Region. Professional Agricultural Workers Journal, 6(1), 24-41.

Lamo, J., Tongoona, P., Sie, M., Semon, M., Onaga, G., \& Okori, P. (2017). Upland Rice Breeding in Uganda: Initiatives and Progress. Advances in International Rice Research, 214-246. https://doi.org/10.5772/66826

Lee, S. M., Hwang, Y. R., Kim, M. S., \& Chung, M. S. (2019). Comparison of Volatile and Non-volatile Compounds in Rice Fermented by Different Lactic Acid Bacteria. Molecules, 24, 1183. https://doi.org/ $10.3390 /$ molecules 24061183

Linares, O. F. (2002). African rice (Oryza glaberrima): History and future potential. PNAS, 99(25), 16360-16365. https://doi.org/10.1073/pnas.252604599

Mahattanatawee, K., \& Rouseff, R. L. (2014). Comparison of aroma active and sulfur volatiles in three fragrant rice cultivars using GC-Olfactometry and GC-PFPD. Food Chemistry, 154, 1-6. https://doi.org/10.1016/ j.foodchem.2013.12.105

Ocan, D., Zhang, R., Odoch, M., Kanaabi, M., Ibanda, A. P., Akwero, A., ... Rubaihayo, P. R. (2019). Relationship between 2-acetyl-1-pyrroline and aroma in Uganda rice populations with Oryza (barthi, glaberrima and sativa) backgrounds. African Journal of Biotechnology, 18(31), 1016-1024. https://doi.org/10.5897/AJB2019.16960

Pachauri, V., Singh, M. K., Singh, A. K., Singh, S., Shakee, N. A., Singh, V. P., \& Singh, N. K. (2010). Origin and genetic diversity of aromatic rice varieties, molecular breeding and chemical and genetic basis of rice aroma. Journal of Plant Biochemistry and Biotechnology, 19(2), 127-143. https://doi.org/10.1007/ BF03263333

Rai, V. P., Singh, A. K., Jaiswal, H. K., Singh, S. P., Singh, R. P., \& Waza, S. A. (2015). Evaluation of molecular markers linked to fragrance and genetic diversity in Indian aromatic rice. Turkish Journal of Botany, 39, 209-217. https://doi.org/10.3906/bot-1405-117

Sansenya, S., Hua, Y., \& Chumanee, S. (2018). The Correlation between 2-Acetyl-1-pyrroline Content, Biological Compounds and Molecular Characterization to the Aroma Intensities of Thai Local Rice. Journal of Oleo Science, 67(7), 893-984. https://doi.org/10.5650/jos.ess17238

Setyaningsih, W., Majchrzak, T., Dymerski, T., Namiesnik, J., \& Palma, M. (2019). Key-Marker Volatile Compounds in Aromatic Rice (Oryza sativa) Grains: An HS-SPME Extraction Method Combined with GCxGC-TOF-MS. Molecules, 24, 4180. https://doi.org/10.3390/molecules24224180

Shanthinie, A., Dilip, K. R., \& Raveendran, M. (2019). Comparative profiling of volatile compounds in the grains of rice varieties differing in their aroma. Electronic Journal Plant Breeding, 10(2), 614-619. https://doi.org/10.5958/0975-928X.2019.00077.2

Sharma, A. K., Singh, T., Patel, A., \& Yadav, R. A. (2018). Influence of integrated nutrient management practices on scented rice (Oryza sativa L.) pertaining to eastern Uttar Pradesh. Journal of Pharmacognosy and Phytochemistry, 7(5), 1448-1453.

Stefano, B., \& Agronomia, I. (2011). Volatile constituents of cooked rice (Oryza sativa L.) (pp. 1-6).

Wakte, A., Zanan, R., Hinge, V., Khandagale, K., Nadaf, A., \& Henry, R. (2016). Thirty-three years of 2-acetyl-1-pyrroline, a principal basmati aroma compound in scented rice (Oryza sativa L.): a status review. Journal of Science of Food and Agriculture, 97, 384-395. https://doi.org/10.1002/jsfa.7875

Wilkie, K., Wootton, M., \& Paton, J. E. (2007). Sensory Testing of Australian Fragrant, Imported Fragrant, and Non-fragrant Rice Aroma. International Journal of Food Properties, 2912, 26-37. https://doi.org/10.1081/ JFP-120022493

Xia, Q., Mei, J., Yu, W. J., \& Li, Y. F. (2017). High hydrostatic pressure treatments enhance volatile components of pre-germinated brown rice revealed by aromatic fingerprinting based on HS-SPME/GC-MS and chemometric methods. Food Research International, 91, 103-114. https://doi.org/10.1016/j.foodres.2016. 12.001

Xu, Y., Ying, Y., Ouyang, S., Duan, X., Sun, H., Jiang, S., Sun, S., \& Bao, J. (2018). Factors Affecting Sensory Quality of Cooked Japonica Rice. Rice Science, 18, 25(6), 330-339. http://doi.org/10.1016/j.rsci.2018. 10.003 


\section{Copyrights}

Copyright for this article is retained by the author(s), with first publication rights granted to the journal.

This is an open-access article distributed under the terms and conditions of the Creative Commons Attribution license (http://creativecommons.org/licenses/by/4.0/). 